\title{
A new approach to Computer-Based Examinations using word documents and spreadsheets
}

Piyapong Khumrin, Ariyaphong Wongnoppavich, Khemmapop Boonploy, Volaluck Supajatura

Department of Physiology, Faculty of Medicine, Chiang Mai University, Chiang Mai, Thailand, 50200 u4507075@hotmail.com

Department of Biochemistry, Faculty of Medicine, Chiang Mai University, Chiang Mai, Thailand, 50200 ariyaphong.w@cmu.ac.th

Department of Physiology, Faculty of Medicine, Chiang Mai University, Chiang Mai, Thailand, 50200 2boonploy@gmail.com

Department of Microbiology, Faculty of Medicine, Chiang Mai University, Chiang Mai, Thailand, 50200 volaluck.supajatura@gmail.com

\begin{abstract}
This paper describes a new approach to computer based testing where lecturers submit questions via word document which is processed to produce an examination, with student results analyzed and reported in a spreadsheet. The overall process starts with lecturers sending question files in word document format via email to the service provider. The questions are passed through the approval process using the editing system and then transferred to the examination system. The examination system directly accesses information from the question files to create a test, which students complete by inserting their answers directly into the spreadsheet file. Finally, the data are analyzed using spreadsheet formulas and the report system sends the results to students' emails. The document based approach helps the system implementation to be simpler and well accepted by the users while consistent with organizational requirements of moving towards electronic data management.
\end{abstract}

\section{Indexing terms/Keywords}

Computer-based examination system; electronic data management; medical education; information technology.

\section{Academic Discipline And Sub-Disciplines}

Computer science; medical education; information technology

\section{SUBJECT CLASSIFICATION}

Software products programming and innovation; electronic examination systems design

\section{TYPE (METHOD/APPROACH)}

System implementation and innovation

\section{Council for Innovative Research}

Peer Review Research Publishing System

\section{Journal: INTERNATIONAL JOURNAL OF COMPUTERS \& TECHNOLOGY}

Vol 12, No.3

\section{editor@cirworld.com}




\section{INTRODUCTION}

Computer based testing (CBT) is commonly used as a tool for student assessment. Several research studies showed that CBT improved student reasoning, and enhanced student interest ${ }^{1}$. The surveys ${ }^{2-4}$ showed that overall teacher attitudes were positive to CBT and globally it is becoming very widely accepted as a valid assessment method. The positive attitudes were explored into specific issues, concluding that CBT was easy to use, faster, consumed less time than written examinations, reduced time for grading, improved the validity of testing, and was easier for statistical analysis. In summary, CBT gains a lot of benefits compared to paper based testing (PBT) with little difference in testing performance ${ }^{5}$ . However, there are some commonly identified problems in CBT such as it is hard to navigate between questions, a student cannot take notes on the questions, and a computer screen can be harder to read ${ }^{7}$.

This project was established under the faculty vision of promoting e-faculty technologies by using innovation to gain more efficiency but use less natural and human resources. According to the statement of purpose, the goal is to develop new approaches to CBT for formative and summative assessment.

Generally, other CBT systems were mainly designed for web application ${ }^{1,8,9}$. Web-based systems have the advantage of managing the system from the server however the security is hard to control. Furthermore, not every user is comfortable using a web application. Also, a web-based system requires close monitoring, regular upgrades, and sustained maintenance. Our new approach addresses these issues by developing a non web-based examination system and instead applying word documents and spreadsheets under a Java software system.

\section{MATERIALS AND METHODS}

The system was implemented at the Faculty of Medicine, Chiang Mai University, Chiang Mai, Thailand. The system was designed to cover the full spectrum of CBT, from receiving examination questions, editing examination questions, storing questions in a library, producing an examination for students to take, to reporting scores and basic statistical analysis. Our target groups are medical students and health science students.

The system requires two basic components: computers with networks; and software. We used Ubuntu 12.04 for the operating system and software written in Java for facilitating the examination system. We adapted an old networked computer system which was previously used for the laboratory demonstration of pathologic section images and the software was totally written by us using open source libraries.

\section{The system contains six sub-systems:}

\section{Preparation system}

First, lecturers prepare examination questions by filling information into tables in the word document templates ${ }^{10}$. We call this template file the "question file". The question file requires general information such as the name of the course, lecturer's name, type of question, level of question (recall, identify, interpretation, apply), the topic corresponding to Thai medical competency assessment criteria for national license, references, and examination information such as question stem, choices, correct answer, and keywords. Each item of information is fixed to be filled in a specific cell in the table because the software selects data according to the corresponding cell position. First, lecturers download the template to prepare the examination contents by themselves. Special characters and text styles have to be written in html format and sub-content is separated by paragraph. Second, the lecturer fills in a list of students' names, identification numbers, emails, and other examination properties into a spreadsheet. We call this spreadsheet the "examination detail sheet". Finally, the question files and examination detail sheet are sent to the examination system assistant's email.

\section{Editing system}

The administrative assistant consolidates the question files and imports them into the editing system. The system generates an identification number for each question file, makes a copy, stores them in the storage directory, and records the question data into a MySQL database. The original contents are kept as the word document files whereas the data in the MySQL database are used only for searching and filtering questions. The lecturers can use the editing system to search for question files, including access to previous examinations on a similar topic. The searching criteria are constrained by question properties. The search results show a list of the names of question files. The users choose a question from the list to view and edit the question in Libre office writer. The editing system monitors the file update while the users are editing questions and simultaneously update the data in the MySQL database referring to the change of data. After all questions are completely edited, the lecturer either uses the editing system to export the questions into one examination sheet for using in PBT, or exports the questions into a question file in CBT.

\section{Examination system}

The system assistant transfers the question files and examination detail sheet into the examination system server. The system assistant starts the examination system to retrieve data from question files and transfer question data to client computers. The master system on the server sends the command triggers to control all client computers, such as executing software, starting the examination, stopping the examination, closing software, and shutting down. When the students select their answer, this is transferred back to the main server and recorded into the examination detail sheet. An optional survey can be included following the test. The system retrieves survey questions from the examination detail sheet and renders these to the client screen after students submit their examination. The results of the survey are sent back to the system and recorded in the questions detail sheet. 


\section{Monitoring system}

The system monitors the progression of students' examinations. Grids on the server screen show a progress bar of how many questions that each student has completed. All trigger events, either from the server or the client, are recorded into another spreadsheet as a log file. The system monitors the action on the main client panel. Several CBT systems minimized the invigilation risk by using accounts to login, identification, real time monitoring, digital certification, data transmission under secure protocol, randomized questions, time limited questions, right click disable. We applied these regular methods, and specially designed two additional features: a full screen mode and deactivation trigger. The panel on a client examination screen was displayed in full screen mode in order to protect the system. A trigger event was added to the panel so that when the panel is deactivated, the panel is automatically locked. Only the administrative assistant can unlock the panel.

\section{Grading system}

The system was designed as an auto-grading system by using spreadsheet formulas. The formulas automatically calculate the number of questions, number of students, maximum score, minimum score, average, standard deviation, minimal passing level, and Kunder-Richardson formula $20^{11}$.

\section{Report system}

A Java application reads information in the spreadsheet, creates a result within an html template and sends the results to student emails. The system required two additional libraries: the Apache POI is used for accessing information from the spreadsheet; and Java mail API ${ }^{12}$ is used for sending examination results to student emails.

\section{Special features of software development and system design}

\section{Access Word document Information by using Apache POI}

Microsoft Word 2007 file format is used as a template for filling question data in the preparation system. The editing and examination systems apply Apache POI to read information from these question files. Apache POI ${ }^{13}$, the Java API for Microsoft documents, is an open source tool for manipulating data in Microsoft Office. In order to get correct information, we created tables in the question file for modeling the structure of data input. The input can be either text or images. The image is simply inserted into the document and set as an anchor as a character. The content in each table cell can be separated into paragraphs for making many question stems and separated keywords.

\section{System security}

The system infrastructure was designed to separate the examination network from the internet connection and the server was placed in a separate room from the examination room. The examination system provides a randomized option for ordering questions and choices in order to increase the difficulty of copying answers. The identification code of each choice is embedded before the choices are shuffled. The system uses hidden identification for scoring without the effect of shuffling. All questions and software are kept in the server and only during the actual examination the data are temporarily transferred to the client computers within a closed secured socket. The results of examinations are kept confidential and individual results are sent to corresponding student emails. The examination system was not designed to have a permanent database on the server. After the examination is finished, the examination results in the spreadsheet are allocated back to the user and nothing is left behind in the server.

\section{Data allocation}

In the client computers, the software does not contain any question contents or even examination software but it works as an initiator using a Java class loader. The Java class loader dynamically loads all necessary classes from the server when the software is initialized. This simplifies the process for doing software updates by replacing an updated version only on the server and protecting examination software from unauthorized persons. The system pulls question information from question files and examination properties from the examination detail sheet. The server creates a set of graphical examination panels containing the examination information and sends the package to the client computer. The client computer converts the input from the server to the graphical examination panels and inserts them into the main panel.

\section{System management for securely saving answers to the spreadsheet}

The limitation of recording answer responses from the examiners to the spreadsheet is that multiple users cannot access the spreadsheet at the same time. Multiple accesses can cause the file to crash and lose data. In a real situation, over a hundred client computers send information to the server for every performance of starting an examination, choosing answers, submitting the examination results, and filling in a survey. Each event is created as a thread and sent independently to the server. Consequently, multiple threads are created while the students are taking the examination, with the very high risk of creating multiple accesses. However, the purpose of running multiple tasks in parallel is to avoid the disruption of the main examination process. In Java programming, the synchronized method and join method help us to organize the multiple data entries to be a single stream line. The methods permit only one thread to do the job and wait until it finishes before the next thread can enter. We applied these methods to make sure that multiple accesses will never happen. 


\section{Using a spreadsheet as a database and analytic tool}

Most CBT systems use a MySQL database for data storage because it is well designed for manipulating data, however it requires a server, security system, well trained assistant, and sustained system maintenance. With our new approach, the system does not require a database for collecting questions. The system was designed to apply a spreadsheet for data management instead. Using the spreadsheet decreases the step of data processing. The data are directly inserted into the cells of the spreadsheet and able to be further analyzed in any computers. The auto-grading system uses existing tools from spreadsheet formulas. The formulas are set before the results are filled and they calculate basic statistical analysis. The results of the examination show immediately after the examination finishes. For example, we used 'IF' spreadsheet function for matching the answer and sum the scores in one cell. Although the new system did not significantly decrease the process of calculating examination results compared to the PBT, the score is ready to use immediately after the examination ends without the need of a specialized score analyzing machine.

\section{Execute remote GUI software}

Basically, we can directly execute software on each client computer. However, it takes long time to launch software on each individual computer especially when we have many computers. Using a combination of SSH and the Expect script language helps us to reduce the workload by sending a remote command from the server to client computers. Each computer must have installed the openssh-server ${ }^{14}$, openssh-client ${ }^{14}$, and expect script library ${ }^{15}$ before using this protocol. The server communicates to client computers over a network using the SSH protocol. The automated login and program executing commands are performed by the Expect script. The Expect script interactively sends requests and passwords to client computers to establish the SSH connection. After the connection is successfully created, the Expect script sends a set of terminal commands to execute the examination software and finally close the connection.

\section{DISCUSSION}

Initially we developed a web-based system according to information from other CBT reviews for building a question database but this was not successful because the users struggled to adapt to the new web-based system. The users were familiar with and preferred to prepare the questions in word document format and send the questions via email. After we received very low attitude feedback from two web-based examination system workshops, we finally decided to cancel the web-based system and use the word document system with the submission by email. We learned from this pitfall that seeking technology to build a system based on user preferences is more effective than forcing users to change.

The new system provides options to create several kinds of questions rather than just plain text. High quality color image is a big advantage for examinations in anatomy, pathology, hematology, parasitology, dermatology, and radiology. Multimedia such as VDO, sound, and animated clips can also be inserted in the questions. The VDO plays an important role in physical examinations, and abnormal signs. The sound can be used for heart murmurs, abdominal sounds, and abnormal breath sounds. However, multimedia options are still in the future plan of project development. At the moment, we focus on the system validity, security and stability issues.

Follow-up surveys about student perceptions of the CBT were performed after three different examinations, with a total of 545 students. The results showed that over $80 \%$ of students were satisfied with the CBT and $73 \%$ of students preferred to use CBT to PBT in a final examination. The percentage of CBT preference was slightly decreased because some students preferred to use CBT as a part of the learning process, for formative assessment, and for small summative assessment.

The first survey showed that students' main complaint about CBT was that it was inconvenient for taking notes. Sometimes, the students wanted to do calculations, draw a mind map, or exclude wrong choices. We discussed this problem and came up with two possible solutions. The first solution was to add a sheet of notepaper for the examination. This solution increases the paper use but it is simple and does not require advanced technology. The second solution was to incorporate using a touch screen and/or pen mouse to take notes on the screen. We could write software to support this option and still keep the paperless goal but we would have to invest for the touch screen and/or pen mouse which is not cost effective at the moment. In the third examination, we added a piece of paper for taking notes. The third survey showed that $73 \%$ of students responded that having notepaper affected the result of the examination. This result implied that the note is helpful but not essential in the process of CBT. Other student complaints were that the computer screen was harder to read and caused tiredness of eye concentration. We could diminish this problem by adjusting background color, font style, and font size.

In summary, our CBT increased privacy, security, data validity, and efficiency of time and data management. The lecturers simply send question files without the additional processes of filling questions onto paper, duplicating papers, handing papers to students, collecting papers from students, ordering answer sheets, sending answer sheets for evaluating results, and grading students. Furthermore, it decreased paper usage and processing and delivery time. For instance, the first formative test with 30 questions and 250 students could reduce paper usage by at least 2,750 sheets.

\section{CONCLUSION}

Applying word documents and spreadsheets under system processing by Java software is a new approach for the CBT system. The existing user familiarity with word documents and spreadsheets is very helpful to implement the system without requiring sophisticated technologies or expensive training. The system is suitable to be applied to other situations where there are conditions requiring non web-based systems, limited computer and programming experts, and a low budget for system implementation. 


\section{ACKNOWLEDGEMENT}

We would like to thank the Tilley family who always give me advice, our team from the IT department who are working very hard to help me implement the system. Most importantly, thank you to the Medical Curriculum unit, Department of Physiology, Faculty of Medicine, and Chiang Mai University for facilitating this project.

\section{FUNDING}

This research received no specific grant from any funding agency in the public, commercial, or not-for-profit sectors.

\section{REFERENCES}

1. Ge $\mathrm{Y}$, Libin H, Lei S, editors. A Web-Based Examination and Evaluation System for Computer Programming. Management and Service Science (MASS), 2010 International Conference on; 2010 24-26 Aug. 2010.

2. Jamila M, Tariqb RH, Shami PA. Computer-based vs Paper-based Examination: Perceptions of University Teachers. The Turkish Online Journal of Educational Technology. 2012;11(4):371-81.

3. Fang Z, Jinhui L, Hongwei J, editors. Design and implementation of computer examination system based on genetic algorithm. Computer Science and Service System (CSSS), 2011 International Conference on; 2011 27-29 June 2011.

4. Fyfe G, Meyer J, Fyfe S, Ziman M, Sanders K, Hill J. Self-evaluation of assessment performance can enhance students' perception of feedback on computer-generated tests.

5. Butler DL. The Impact of Computer-Based Testing on Student Attitudes and Behavior. The Technology Source. 2003.

6. Lim EC, Ong BK, Wilder-Smith EP, Seet RC. Computer-based versus pen-and-paper testing: students' perception. Annals of the Academy of Medicine, Singapore. 2006;35(9):599-603. Epub 2006/10/20.

7. Jimoh RG, andKawu AKS, Kola Y. Students' Perception of Computer Based Test (CBT) for Examining Undergraduate Chemistry Courses. Journal of Emerging Trends in Computing and Information Sciences. 2012;3(2):125-34.

8. Oluwatosin OT, Samson DD. Computer-Based Test: Security and Result Integrity. International Journal of Computer and Information Technology. 2013;2(2):324-9.

9. Liang Z, Yue-ting Z, Zhen-ming Y, Guo-hua Z, editors. A Web-Based Examination and Evaluation System for Computer Education. Advanced Learning Technologies, 2006 Sixth International Conference on; 2006 5-7 July 2006.

10. Curriculum Management Unit. Faculty of Medicine, Chiang Mai University; 2013 [updated 14 October 2013; cited 2013 1 October 2013]; Available from: http://www.med.cmu.ac.th/secret/edserv/curriculum/.

11. Kuder GF, Richardson MW. The theory of the estimation of test reliability. Psychometrika. 1937;2(3):151-60.

12. Foundation TAS. Commons Email. 2012 [updated 3 March 2013; cited 20131 September]; 1.3.1:[Available from: http://commons.apache.org/proper/commons-email/.

13. Oliver AC, Stampoultzis G, Sengupta A, Klute R, Fisher D. Apache POI - the Java API for Microsoft Documents. The Apache Software Foundation; 2013 [cited 20131 October 2013]; Available from: http://poi.apache.org/.

14. OpenSSH Server. 2013 [updated 19 July 2013; cited 20131 October 2013]; Available from: https://help.ubuntu.com/12.04/serverguide/openssh-server.html.

15. Libes D. The Expect Home Page. The National Institute of Standards and Technology (NIST) 2009 [updated 3 August 2009; cited 20131 October 2013]; Available from: http://expect.sourceforge.net/.

\section{Author' biography with Photo}

\begin{tabular}{|l|l|} 
Dr Piyapong Khumrin is a full time lecturer at the Department of Physiology, Faculty of \\
Medicine, Chiang Mai University, Chiang Mai, Thailand. He graduated Doctor of \\
Medicine in 2008. One year later, he continued his graduate study and finished \\
Master of Science in Engineering specialized in Medical Informatics in Austria. After \\
he finished his Master degree in 2011, he is leading several research projects such as \\
the implementation of integrated heterogeneous electronic ECG data into Maharaj \\
Nakorn Chiang Mai hospital information system, electronic scoring system for small \\
group discussion, electronic examination system with the Faculty, e-learning and \\
multimedia teaching tools for students. His scopes of interest are health \\
interoperability, electronic health records, information ontology, information \\
technology, database management, system integration, health information \\
standardization, clinical decision making, expert system, telemedicine, e-learning, and \\
medical education. He is looking forward to study PhD in clinical decision making in \\
medical time-series data.
\end{tabular}




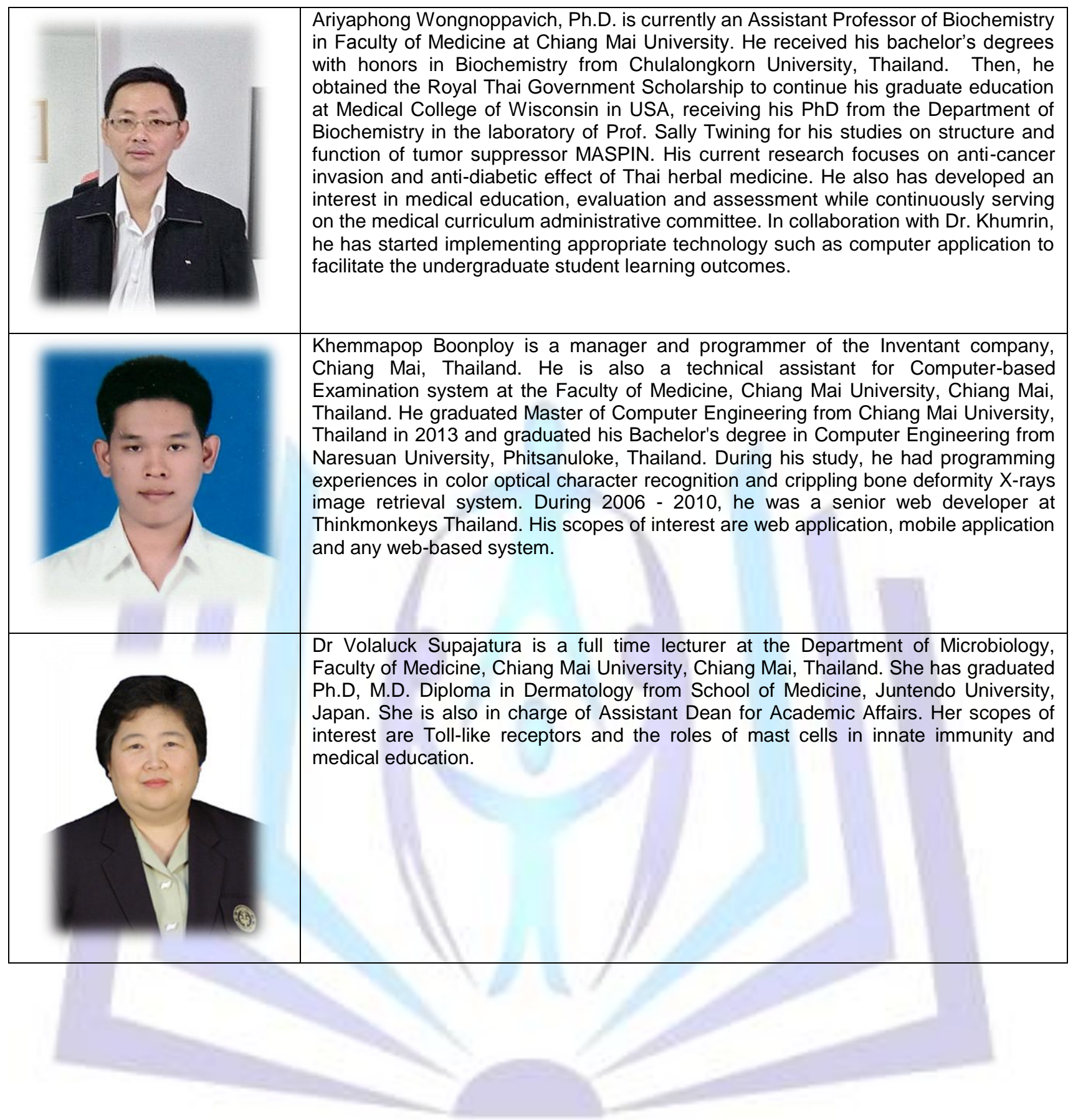

